\title{
Design and Realization of an Integrated Optical Frequency Modulation Discriminator for a High Performance Microwave Photonic Link
}

\author{
D.A.I. Marpaung, C.G.H. Roeloffzen, R.B. Timens \\ Telecommunication Engineering group \\ University of Twente, PO Box 217, 7500 AE \\ Enschede, the Netherlands \\ email: d.a.i.marpaung@ewi.utwente.nl
}

\author{
A. Leinse, M. Hoekman \\ LioniX BV \\ PO Box 456, $7500 \mathrm{AH}$ \\ Enschede, the Netherlands
}

\begin{abstract}
This paper reports the design, fabrication and the characterization of an integrated optical filter for an FM discriminator. The filter is based on optical ring resonator structures which are fully reconfigurable using thermo-optical tuning. The desired characteristic, which is a linear slope with zero in a particular region, is demonstrated. This characteristic is needed in a high performance microwave photonics link with increased spurious free dynamic range.
\end{abstract}

\section{INTRODUCTION}

High performance microwave photonic links (MPLs) are attractive for a host of applications like antenna remoting, radio over fiber and phased-array antenna. To serve these applications the MPLs need to fulfill several criteria namely high link gain, low noise figure and high spurious-free dynamic range (SFDR). High SFDR dictates high linearity and low noise in the MPLs. In an intensity modulated direct detection (IMDD) MPL the SFDR is limited mainly by the laser relative intensity noise (RIN) and the third order intermodulation distortion (IMD) either from the laser or the electro-optic modulator (EOM). In such IMDD links, the SFDR can be increased in several ways. An MPL with a Mach-Zehnder EOM benefits from low biasing the EOM that reduces the link noise, while an MPL with directly modulated lasers might achieve high SFDR using balanced push-pull architecture that removes even-order IMDs [1]. Recently significant interest is directed towards the SFDR enhancement in a different type of MPL called the frequency-modulated direct detection (FMDD) links. In such links, a frequency modulated signal is converted to intensity modulation using an optical filter, thereby allowing a simple direct detection scheme instead of the complicated coherent detection. The interest in such a scheme stems from two reasons; first, frequency modulation of a laser has the potential of high modulation efficiency, bandwidth and linearity [2] and second, there is an additional degree of freedom in tailoring the characteristic of the optical filter discriminator to enhance the MPL performance. Thus, in such an approach, the FM discriminator is designed for increasing the link linearity and/or suppressing the noise in the MPL. In previously reported investigations, different filter types have been proposed as the FM discriminator [2-5]. The simplest one, being a Mach-Zehnder interferometer (MZI), suffers from large nonlinearities [2]. Fiber Bragg-gratings (FBGs), have also been widely considered for the FM discriminator, notably with the potential of noise reduction [3]. However these FBGs and the circulators needed for such solutions are bulky and thus, preventing a compact discriminator. Integrated optics solutions have also been proposed $[2,4,5]$ but, to our knowledge, these are limited to concept and have not been realized yet.

In this paper, for the first time to our knowledge, the design, realization and characterization of an integrated optical filter for an FM discriminator are reported. The filter consists of optical ring resonators (ORRs) which are fully reconfigurable using thermo-optical tuning. The filter transfer function is designed and adjusted to reduce the noise and nonlinearities in the FMDD MPL to maximize the SFDR. The rest of the paper is organized as follows: In Section II the MPL principle of operation and the desired filter structure is discussed. The proposed filter structures are explained in Section III. The filter fabrication and packaging processes are described in Section IV. In Section $V$, the filter characterization results are presented. The paper closes with conclusions.

\section{THE LINK ARCHITECTURE AND THE DESIRED FILTER RESPONSE}

The architecture of the FMDD MPL is shown in Fig. 1. The modulated optical signal from an FM laser is routed to the receiver site using a single optical fiber. The receiver consists of the FM discriminator optical chip and the balanced photodetector (BPD). The filter has one optical input and two optical outputs where the ideal transfer functions from the input to the two outputs are mirror images of each other, as shown in Fig.1. The transfer function has a sharp transition at the angular frequency of the optical carrier $\left(\omega_{\mathrm{c}}\right)$. Ideally, at

This work is a part of the European FP7 SANDRA project. 


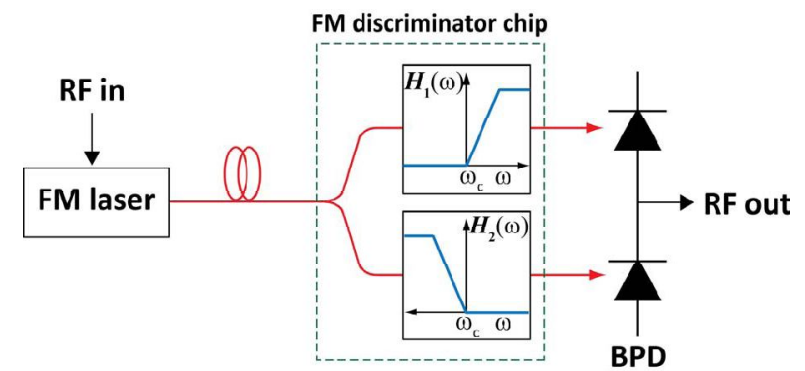

Figure 1. Schematic of the FMDD microwave photonic link

the upper branch, for frequencies below $\omega_{\mathrm{c}}$ the magnitude filter response is zero while above the $\omega_{\mathrm{c}}$ it is linear up to a maximum frequency where the filter transfer is flat at the maximum transfer. This also applies to the other output for frequencies below $\omega_{\mathrm{c}}$. Supposed that the carrier frequency is modulated with an RF sinusoidal signal, the resulting instantaneous frequency above $\omega_{\mathrm{c}}$ will be converted by the upper filter to intensity fluctuations while frequencies below $\omega_{\mathrm{c}}$ will be attenuated. Thus, the intensity profiles at the filter outputs resemble complementary half-wave rectified optical signals. These signals will then be subtracted in the BPD restoring the RF signal. The half-wave rectification will yield to substantial shot noise and RIN reductions in the MPL [3]. It is thus crucial to maintain the linearity of the filter transfers such that the noise reduction can be translated into an SFDR enhancement of the MPL. An MPL with these characteristics is called the class-B MPL, initially proposed by Darcie et al. in [3].

In order to realize the desired filter, an optical ringresonator (ORR) has been chosen in this work as the building block. The ORR considered here is a racetrack structure connected with a pair of tunable couplers to two straight optical waveguides, as depicted in the inset of Fig. 2. The reason to choose this structure as the building block is that the drop response of such an ORR already bears a resemblance of the desired filter curve. This is depicted at Fig. 2, where the simulated drop and through responses of an ORR are shown. Here a frequency scale normalized to the free-spectral range (FSR) of the ring has been used. In the simulations, several parameters of the ORRs can be varied. These are the optical loss per round trip in the ORR, the resonance frequency and the coupling coefficients $\left(\kappa_{1}\right.$ and $\left.\kappa_{2}\right)$ that determine the Qfactor of the ORR.

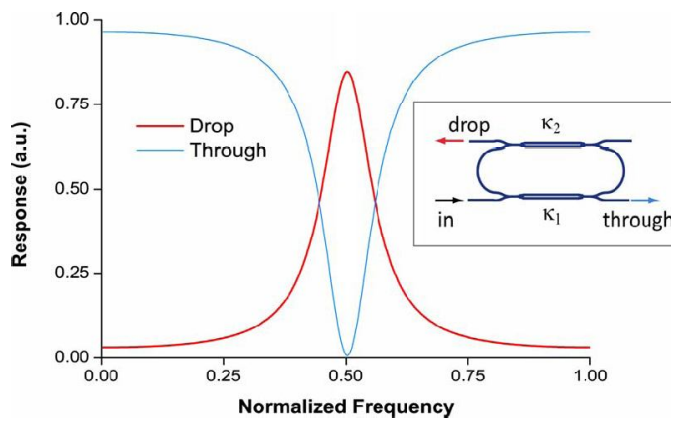

Figure 2. The drop and through responses of the ORR building block (inset)

\section{FILTER DESIGNS}

There are two filter structures proposed in this work. The first structure contains three ORRs with the same FSR (3-ORRs) while the second structure is the extension of the first structure where two ORRs with half the FSR (thus twice the circumference) of the original rings are added (5-ORRs). The schematic of the 3-ORRs and 5-ORRs designs are shown in Fig. 3 (a) and (b) respectively.
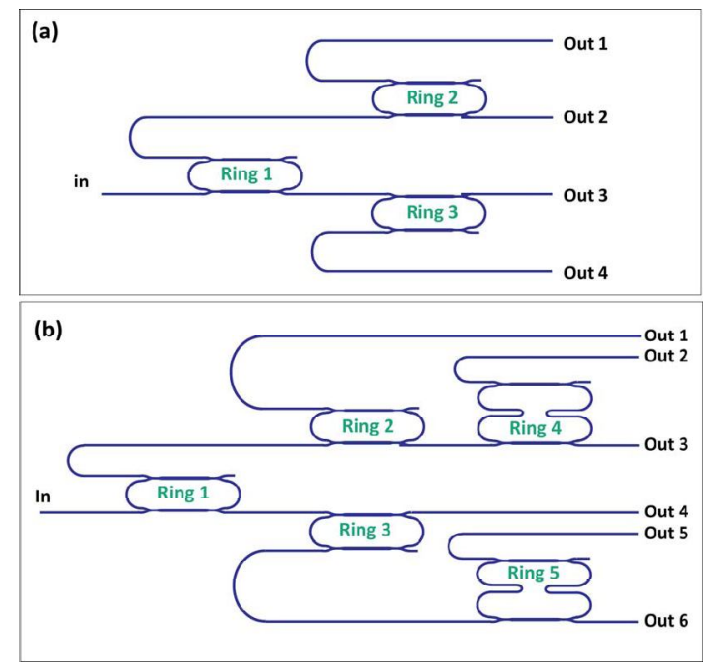

Figure 3. The 3-ORRs (a) and the 5-ORRs (b) filter designs

\section{A. Three-ORRs design}

As mentioned earlier, the drop response of an ORR resembles the desired response of the ideal FM discriminator. However, this response is lacking the suppression in the region where the ideal response goes to zero. Thus in the 3-ORRs design, this suppression is enhanced by cascading the drop response of the ORR with a through response of another ORR, where the resonance frequencies of these ORRs are slightly detuned. In the 3-ORRs design (Fig. 3(a)), the drop response of ring 1 is cascaded with the through response of ring 2 to yield one of the desired outputs, while at the same time the through response of ring 1 is cascaded with the drop response of ring 3 to yield the other desired output. This is illustrated in Fig.4 (a) where the simulated responses of the 3-ORRs structure are depicted.

\section{B. Five-ORRs design}

As evident from Fig. 4(a), adding a through response of an ORR to the drop response will improve the suppression in the desired filter response. However, the 3-ORRs response is still lacking the sharp transition region in the ideal filter response. For this reason the 5-ORRs filter design is introduced. As shown Fig. 3 (b), in this design, two ORRs with half of the FSR of the original rings are added to each of the desired output ports. The resonance frequencies of these ORRs are finely tuned between the resonance frequencies of the drop and the through responses of the 3-ORRs configurations such that the sharp transition regions are achieved. This is illustrated in Fig. 4(b). 

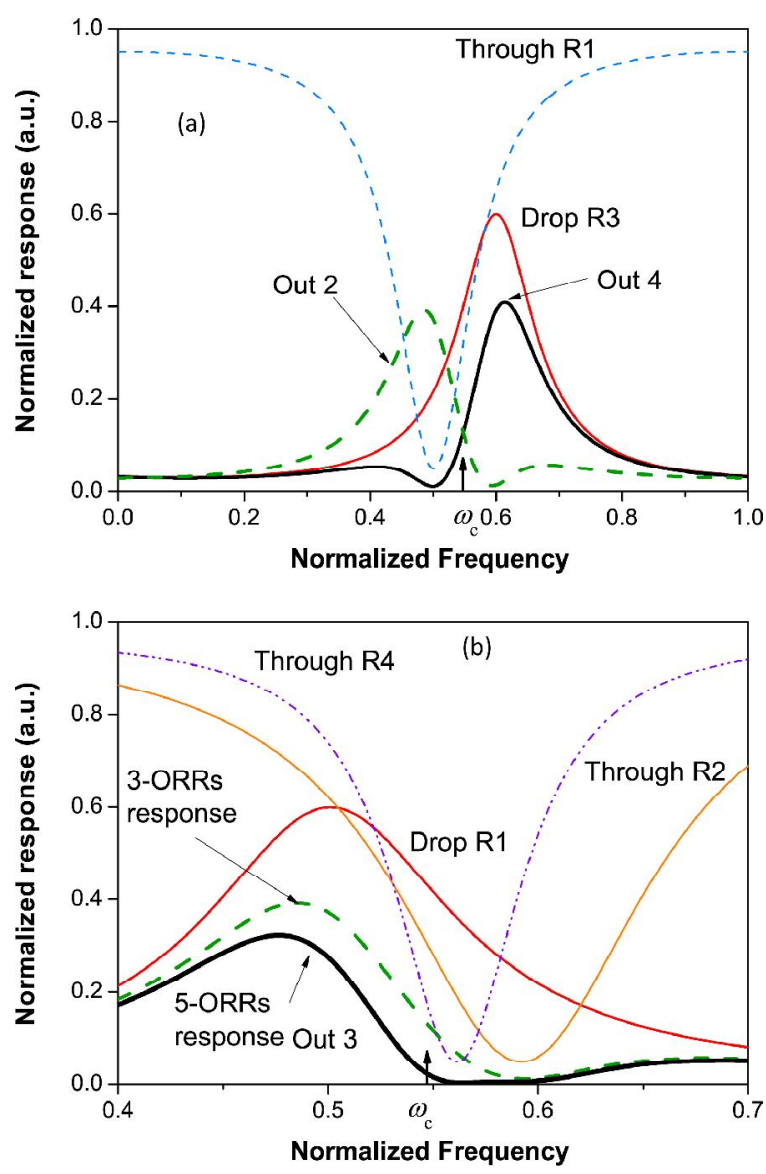

Figure 4. Simulated responses for the 3-ORRs (a) and the 5-ORRs (b) filter designs

\section{FABRICATION AND PACKAGING}

The designed 3-ORRs and the 5-ORRs filters are fabricated in the TriPleX ${ }^{\mathrm{TM}}$ waveguide technology with a high contrast box shaped waveguide structure [6]. The round trip length of the rings is $8 \mathrm{~mm}$ ( $16 \mathrm{~mm}$ for rings 4 and 5) and the radius of the curved part of the ORR is $150 \mu \mathrm{m}$. The total chip footprint is $9 \times 7 \mathrm{~mm}$. The resonance frequency and the coupling coefficients of every ORR are fully tunable by means of thermo-optical tuning. For this tuning, chromium heaters are deposited on the chip. The optical layout of the 3-ORRs and the 5-ORRs chips are shown in Fig. 5 (a) and (b), respectively. The detail of the chip fabrication and characterizations will be published elsewhere.

For the ease of measurements, the fabricated chips are packaged. The bondpads for the heaters are wirebonded to a pair of PCBs. An array of 8 polarization maintaining fibers are aligned and glued to the inputs of the optical chip while at the output the chip is pigtailed with an array of standard single-
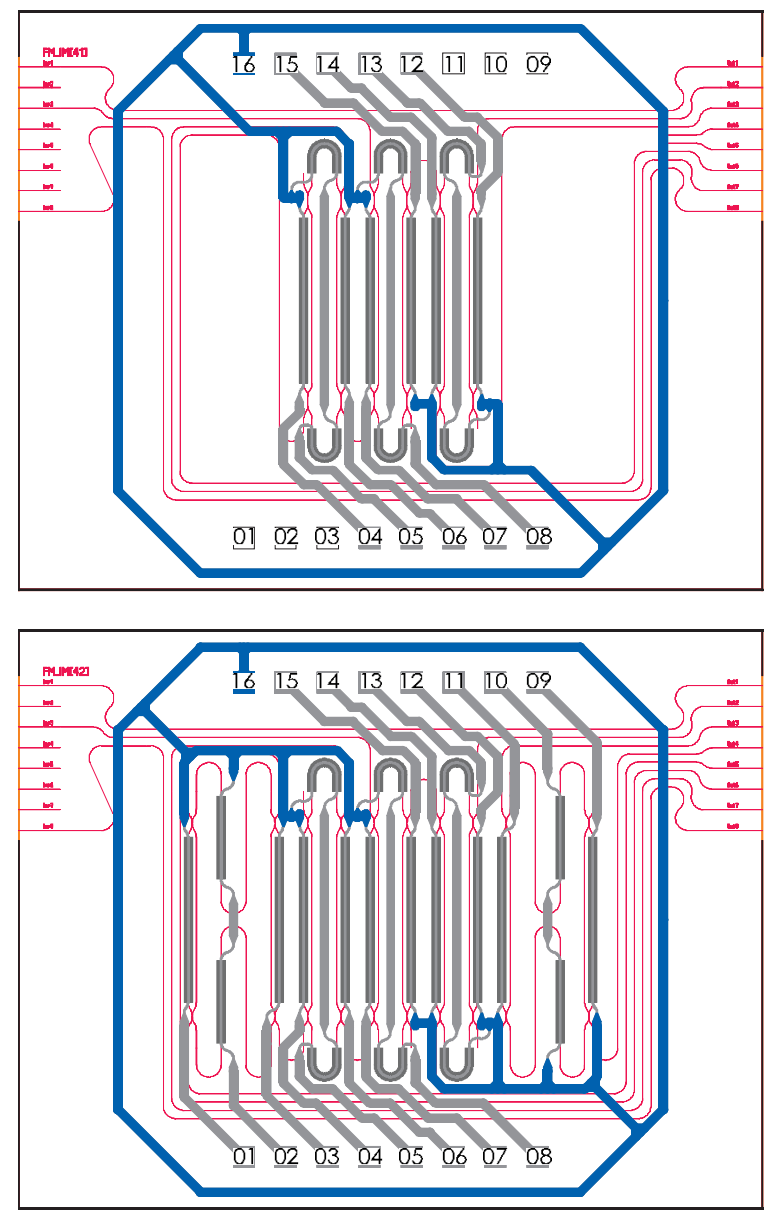

Figure 5. The waveguide layouts and the heater configurations for the 3-ORRs (upper) and the 5-ORRs (lower) filter designs

mode fibers. For the desired outputs (outputs 2 and 4 for the 3-ORRs chip and outputs 3 and 6 for the 5-ORRs chip) high numerical aperture (HNA) fibers are spliced to the SMFs to reduce the effect of stray light in the chip. The photograph of the packaged 5-ORRs FM discriminator is shown in Fig. 6.

\section{CHARACTERIZATIONS}

The characterizations were performed on the packaged 5-ORRs chip only since with proper tuning the response of 3-ORRs filter can also be obtained from the 5-ORRs chip. In this chip a test waveguide containing a straight part and two bends is fabricated to check the propagation loss of the optical waveguides. A measurement on this waveguide reveals a $25 \mathrm{~dB}$ insertion loss of the optical chip. This loss is mainly attributed to the fiber-to-chip coupling loss, where there is no spot-size converter implemented either in the input or the output. We then proceed with the characterization of the filter responses. In order to configure the rings, a heater controller is used to supply the voltages to the heaters. 


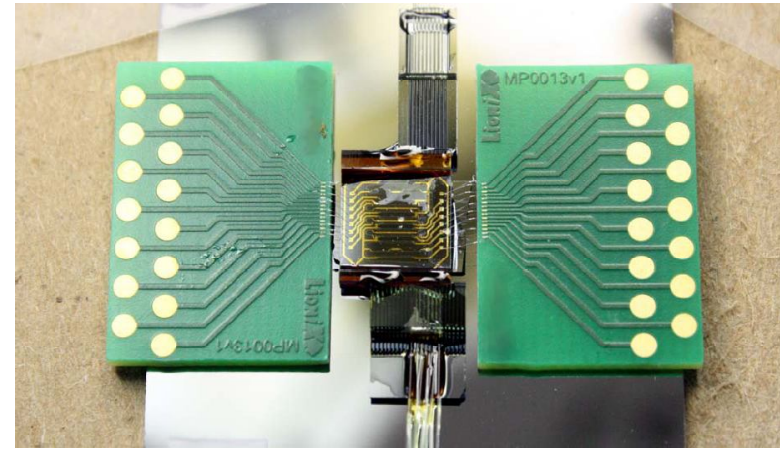

Figure 6. Photograph of the packaged 5-ORRs chip

Currently the controller is capable of controlling a maximum of 12 channels. Since three heaters are needed to fully tune the resonance frequency and the $\mathrm{Q}$ factor of a ring, a maximum of 4 rings can be configured simultaneously. This is not enough to fully configure the 5-ORRs filter and to obtain the two desired responses from the two outputs. For this reason, the measurements presented here are limited to one of the desired responses. We have chosen to configure rings 1,3 and 5 in the chip (Fig. 3(b)). Initial measurements were performed on output 4 of the chip. By tuning rings 1 and 3 , the output optical power from this output can be maximized. This measured optical power is then used to normalize all measurements presented here. Next, ring 3 is tuned out of resonance and ring 1 is tuned in resonance to yield the through response of ring 1 , which is shown as the dotted curve in Fig.7 (a).

The drop response of ring 3 is measured from output 6 of the chip by tuning ring 3 in resonance and tuning rings 1 and 5 out of resonance. The result is shown as the solid thin line in Fig. 7(a). The 3-ORRs response is then obtained from this output by tuning both rings 1 and 3 on and turning off ring 5, and by subsequently adjusting the distance of the rings resonance frequency. The result is shown in the same figure as the solid thick line. The measured response agrees very well with the simulated response shown in Fig. 4(a). Furthermore, starting from this response, ring 5 is then tuned in-resonance (solid thin curve of Fig. 7(b)) and the resonance frequency is tuned such that it is positioned in-between the resonance frequencies of rings 1 and 3 to yield the 5-ORRs response (dash-dotted line of Fig. 7(b)). This response also agrees with the expected response in Fig. 4(b). We also have shown that the 5-ORRs response is improved in terms of suppression and linearity in the transition region. The fact that the 5-ORRs magnitude response is significantly lower compared to the drop response of a single ORR stems from the propagation loss in the optical waveguide.

\section{CONCLUSIONS}

In this paper the design, fabrication and characterization of an integrated optical FM discriminator have been reported. The principle of cascading the drop response of an ORR with multiple through responses of other ORRs to yield a desired
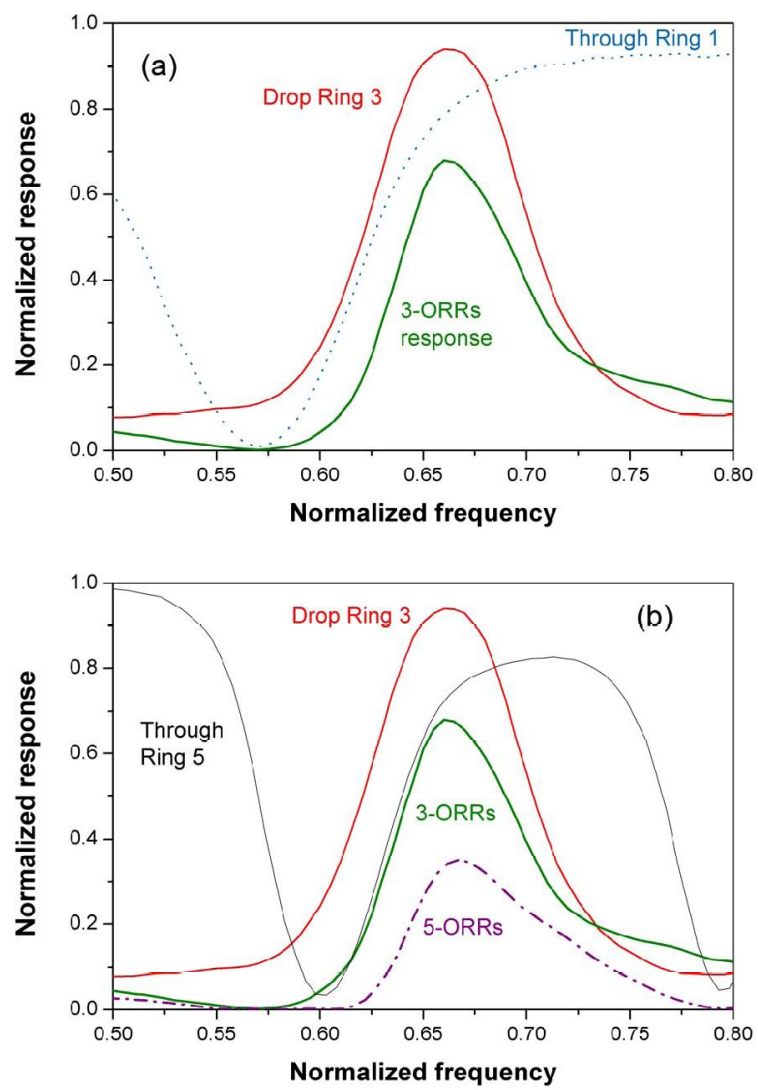

Figure 7 . Measured responses for the 3-ORRs (a) and the 5-ORRs (b) filters

response with a sharp transition between suppressed region and a linear slope has been demonstrated. The subsequent steps are to fully configure the complementary responses of the filter, to implement the discriminator in an FMDD MPL and to characterize the performance of such an MPL.

\section{REFERENCES}

[1] D. Marpaung, C. Roeloffzen, and W. van Etten, "Enhanced dynamic range in a directly modulated analog photonic link," IEEE Photon. Technol. Lett., 21(24), pp. 810-812, Dec. 2009.

[2] J. Wyrwas and M. Wu, "Dynamic range of frequency modulated direct-detection analog fiober optic link," J. Lightw. Technol., 27(24), pp. 5552-5562, Dec. 2009.

[3] T.Darcie, J. Zhang, P. Driessen and J. Eun, "Demonstration of a class-B microwave-photonic link using optical frequency modulation and complementary fiber-Bragg grating discriminators", OFC/NFOEC, Anaheim, CA, 2006, postdeadline paper P38.

[4] X. Xie, J. Khurgin, J. Kang, and F. Choa, "Compact linearized optical FM discriminator," IEEE Photon. Technol. Lett., 14(3), pp. 384-386, Mar. 2002.

[5] X. Xie, J. Khurgin, J. Kang, and F. Choa, "Ring-assisted frequency discriminator with improved lineasriy," IEEE Photon. Technol. Lett., 14(8), pp. 1136-1138, Aug. 2002.

[6] F. Morichetti et al, "Box-shaped dielectric waveguides: A new concept in integrated optics?," J. Lightw. Technol., 25(9), pp. 2579-2589, Sep. 2007. 